\title{
Bluetooth ACL Packet Selection Via Maximizing the Expected Throughput Efficiency of ARQ Protocol
}

\author{
Xiang $\mathrm{Li}^{1,2, *}$, Man-Tian $\mathrm{Li}^{1}$, Zhen-Guo $\mathrm{Gao}^{2}$, and Li-Ning $\operatorname{Sun}^{1}$ \\ ${ }^{1}$ Robot Research Institute, Harbin Institute of Technology, Harbin, 150001, China \\ ${ }^{2}$ College of Computer Science and Technology, Harbin Engineering University, Harbin, \\ 150001, China \\ \{leexiang, gag\}@hrbeu.edu.cn, \{limt, lnsun\}@hit.edu.cn
}

\begin{abstract}
Bluetooth provides different kinds of data packet types with different sizes and error correction mechanisms, thus adapter layer can choose the most suitable packet to be transmitted according to the error rate on the link and application requirements. Based on the acknowledgement history of the most recently transmitted packets, an adaptive algorithm is proposed to choose the suitable Bluetooth data packet for transmission through maximizing the expected throughput efficiency of ARQ protocol on Bluetooth ACL data communication link. Simulation results indicate that this method works very well with a short observation history and also show special performance of DM and DH data packet transmission.
\end{abstract}

Keywords: Bluetooth, Piconet, ARQ, ACL, Throughput Efficiency.

\section{Introduction}

Bluetooth (BT) [1,2] is a short-range radio link intended to be a cable replacement between portable and/or fixed electronic devices. Two types of transmission links, SCO and ACL links are used. SCO link is a symmetric point to point link supporting time-bounded voice traffic. SCO packets are transmitted over reserved intervals without being polled. ACL link is a point to multipoint link between master and all slaves in the piconet and can use all the remaining slots of the channel not used for SCO link. Bluetooth is a frequency hopping system which can support multiple communication channels in a common area (each channel is defined by a unique frequency hopping sequence). Frequency hopping is used in such a way that the radio is turned to the same frequency for the entire duration of the packet, but then changes to a different frequency each time it transmits a new packet or retransmits an erroneous packet. Since the fading and interference in the new frequency channel will be significantly different than that of the previous one, the use of frequency hopping with ARQ provides an effective method of diversity.

Automatic Repeat Request (ARQ) protocols are designed to remove transmission errors from data communications systems. When used over relatively high bit-error rate (BER) links (e.g., $10^{-5}$ or higher) such as wireless or satellite links, their performance is

\footnotetext{
* Supported by the Harbin Engineering University Foundation (HEUFT06015).
} 
sensitive to the packet size used in the transmission. When too large a packet size is employed, there is an increased need for retransmissions, while too small a packet size is inefficient because of the fixed overhead required per packet. When an ARQ scheme is to be used at the link layer over a relatively high error-rate link, the packet size should be chosen based on the error-rate [3].

The optimal communication problem in Bluetooth has been investigated in some documents. In [4], a solution is proposed to enhance the Bluetooth link layer to make use of channel state information and adopt the suitable Bluetooth packet type to enhance TCP throughput. The throughput of the six Bluetooth ACL packets that use ARQ as a function of channel symbol SNR are derived in [5], then optimal packet type can be selected at different SNRs. Document [6] provides algorithms to maximize the throughput under lossy transmission conditions in a piconet with one or more slaves by selecting the packet lengths optimally in accordance with the channel conditions for different frequencies. All these works are concentrating on throughput under different channel conditions, such as BER or SNR, which are not easy to know ahead. In this paper, we concern with choosing optimal packet payload length on the Bluetooth ACL data communication links, in terms of maximizing the throughput efficiency of ARQ protocol based on the acknowledgement history of the most recently transmitted packets. That is, given the number of packets that required retransmission, an estimate of the channel BER is made, based on which a packet size is chosen to maximizes the expected throughput efficiency of the data link protocol.

\section{Bluetooth Data Packets}

In Bluetooth, the data on the piconet channel is conveyed in packets. The general packet format is shown in Fig.1. Each packet consists of 3 entities: the access code, the header, and the payload. In Fig. 1, the number of bits per entity is indicated [1].

\begin{tabular}{|l|l|l|}
\hline Access Code(72) & Header(54) & Payload(0-2745) \\
\hline
\end{tabular}

Fig. 1. Standard Packet Format

The access code and header are of fixed size: 72 bits and 54 bits respectively. The payload length can range from zero to a maximum of 2745 bits. Different packet types have been defined. Packets may consist of the (shortened) access code only, of the access code - header, or of the access code - header - payload.

Data in Bluetooth can be transmitted asynchronously using ACL packets. In this paper, we mainly focus on ACL packets data transfer used in asynchronously connections. Seven ACL packet types are defined in the Bluetooth. DM stands for DataMedium rate, DH for Data-High rate. DM packets are all 2/3-FEC encoded to tolerate possible transmission errors. Not encoded by FEC, DH packets are more errorvulnerable, but it can carry more information. 


\section{Adaptive Packet Selection Algorithm}

\subsection{Throughput Efficiency of ARQ Protocol}

A protocol performance is usually characterized by many parameters which are defined by the communication system requirements. The most important parameters are the probability of receiving a message without errors and the protocol throughput efficiency. There are several definitions of the protocol throughput efficiency. Most frequently it is defined as the ratio of the mean number of information bits successfully accepted by the receiver to the number of bits that could have been transmitted during the same time interval [7]. To do so we must first derive an expression for the throughput efficiency of the ARQ protocol. The expressions derived in this section assume the use of an "optimal" ARQ protocol in that only packets containing errors are retransmitted. The throughput efficiency of ARQ scheme that uses packets having $\mathrm{n}$ bits of information bits $k$ is determined by [8]:

$$
\eta=\left(\frac{k}{n}\right) / \bar{R}
$$

where, the first term $\mathrm{k} / \mathrm{n}$ of the above expression represents the ratio of information bits to total bits in a packet, and $\bar{R}$ represents the average number of transmission attempts per packet.

Assuming that the ARQ scheme retransmits a packet until the acknowledgement of a successful reception, the average number of attempts, $\bar{R}$, needed to successfully transmit one packet is given by [4]:

$$
\bar{R}=1 \times(1-p)+2 \times p \times(1-p)+3 \times p \times p \times(1-p)+\ldots=\frac{1}{1-p}
$$

where, $p$ is the packet error rate.

So, for a given $p$, the throughput efficiency of ARQ that uses packets having $n$ bits of information bits $k$ is given by:

$$
\eta=\left(\frac{k}{n}\right)(1-p)
$$

\subsection{Choosing Packet Size Via Maximizing the Expected Throughput Efficiency of ARQ Protocol}

When a perfect retransmission algorithm (A perfect retransmission algorithm is one that only retransmits packets that are in error and can continuously transmit new packets as long as no errors occur. The selective repeat protocol is an example of a perfect retransmission algorithm) is employed, the optimal packet size to be used by the data link protocol is given by [3]:

$$
k_{\text {opt }}=\frac{-h \ln (1-b)-\sqrt{-4 h \ln (1-b)+h^{2} \ln \left(1-b^{2}\right)}}{2 \ln (1-b)}
$$

where $b$ is the known channel BER and $h$ is the number of overhead bits per packet(These bits are used for control, error detection, and framing). 
When $h$ equals to 126 , the optimal packet size under different channel BER can be displayed in Fig. 2 by formula (4). Fig. 2 shows that the optimal packet size decreases according to the increase of the channel BER. This change trends meets to the real application requirement because when a much larger packet size is used the efficiency of the protocol would drop dramatically while the channel BER is much higher. Therefore a much smaller packet size is efficient under a much higher channel BER because a small packet has low packet error rate. On the contrary, a much larger packet size makes efficient use of the channel when the channel BER is much lower.

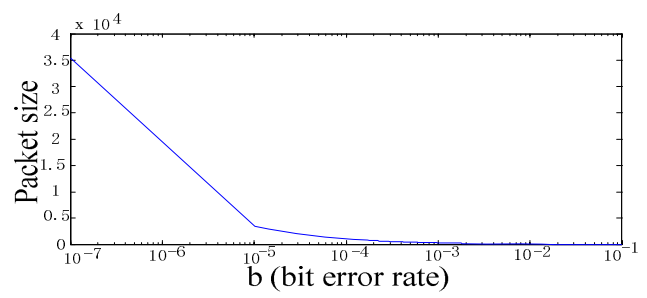

Fig. 2. Optimal packet size under different channel bit error rate

In Bluetooth, in order to guarantee reliable transmission ARQ mechanism is adopted. That is, the receiving side sends back special control frame as the acknowledgement or negative acknowledgement (ACK/NACK) to the input. In case of drop frame or acknowledgement message, the timer will send out timeout signal when the timer has expired, and to remind other sides that some problems have happened and this frame must be retransmitted. At the same time, receiver must be capable of distinguishing between retransmitted and new frame. With an ARQ scheme in Bluetooth specification, DM, DH and the data field of DV packets are transmitted and retransmitted until acknowledgement of a successful reception is returned by the destination (or timeout is exceeded). The acknowledgement information is included in the header of the return packet, so-called piggy-backing. To determine whether the payload is correct or not, a CRC code is added to the packet. The ARQ scheme only works on the payload in the packet (only that payload which has a CRC). The packet header and the voice payload are not protected by the ARQ.

Depending on the packet retransmission records on the current link, an adaptive method to select the best packet for data transmission is proposed to improve the performance of Bluetooth system. The basic idea behind this scheme is that a large packet has low overheads and is advantageous when the BER is relatively low, while a small packet has a low packet error rate and thus is advantageous when the BER is high. So, depending on channel BER, every type of Bluetooth ACL data packet has different performance. Without any bit error, the DH5 packet would give the best performance since it carries the most information bit per unit time. However, as the BER increases, the packet error rate of DH5 increases faster than smaller packets. Thus, there exists a problem to how to select suitable packet to adapt to the current channel conditions. However, it is difficult to estimate the channel BER in a short time when the link is operating under error detection with retransmission [9], but we can acquire the packet retransmission history easily. 
This paper proposes a simple algorithm to choose the packet size such that the conditional efficiency of the protocol is maximized under different channel BERs based on packet transmission record. Supposed the BER is $b$, and $R$, the number of retransmission requests out of the last $M$ packet transmissions, by averaging the above expression over all possible values of $\mathrm{b}$ and using the conditional distribution of $\mathrm{b}$ given $\mathrm{R}$ (assuming that $\mathrm{b}$ is constant over the period of interest). The resulting expression is given by [3]:

$$
\eta_{R}(k)=\int_{0}^{1} \eta P(b \mid R) d b
$$

where $P(b \mid R)$, is the conditional probability of $b$ given that $R$ out of the last $M$ packets required retransmission. We now wish to choose the value of $k$ that maximizes $\eta_{R}$. To do so we must first express the conditional probability of $b$ given $R$.

The conditional probability of $b$ given $\mathrm{R}, \mathrm{P}(\mathrm{b} \mid \mathrm{R})$, can be expressed as follows:

$$
P[b \mid R]=\frac{P[b, R]}{P[R]}=\frac{P[R \mid b] P[b]}{P[R]}
$$

Solving for the above conditional probability requires knowledge of a prior distribution of $b$. In the absence of a prior, we assume a uniform prior, that is $P[b]=1$. This approach, in essence, is the same as a maximum likelihood approach where a uniform prior is assumed, except that here we associate a cost function with the estimates of $b$. With this approach we get

$$
P[R]=\int_{0}^{1} P[R \mid b] P[b] d b=\int_{0}^{1} P[R \mid b] d b
$$

and so

$$
P[b \mid R]=\frac{P[R \mid b]}{\int_{0}^{1} P[R \mid b] d b}
$$

In wireless communication, we generally assume that channels are independent and same distribution model, that is, assume that error rates on the channel are independent of each other and commonly kept invariable. Given packet error rate $p$, the probability that $R$ packets contain errors and therefore require retransmission is the probability that $R$ out of $M$ packet are in error. Since packet errors are independent from packet to packet, this probability can be expressed according to the binomial distribution with parameter $p$, therefore $P[R \mid b]$ can be expressed as:

$$
P[R \mid b]=\left(\begin{array}{c}
M \\
R
\end{array}\right) p^{R}(1-p)^{M-R}
$$

The packet error rate, $\mathrm{p}$, for $\mathrm{DH}$ packets is:

$$
p=1-(1-b)^{k}
$$

Recalling that DM packets are protected by a $(15,10)$ Hamming code (encoded with a $2 / 3$ block FEC), i.e., in every block, 15 bits are used to encode 10 bits of data, which is capable of correcting one bit error per 15 bit code block. The payload is correctly decoded provided that all code blocks contain one or fewer errors. The packet error rate, $p$, for DM packets can be approximated as: 


$$
p=1-\left((1-b)^{15}+15 b(1-b)^{14}\right)^{k / 15}
$$

Hence, for DH packets, $P[R \mid b]$ can be expressed as:

$$
P[R \mid b]=\left(\begin{array}{c}
M \\
R
\end{array}\right)\left(1-(1-b)^{k^{\prime}}\right)^{R}(1-b)^{k^{\prime}(M-R)}
$$

For DM packets, $P[R \mid b]$ can be expressed as:

$$
P[R \mid b]=\left(\begin{array}{c}
M \\
R
\end{array}\right)\left(1-\left((1-b)^{15}+15 b(1-b)^{14}\right)^{k^{\prime} / 15}\right)^{R}\left((1-b)^{15}+15 b(1-b)^{14}\right)^{k^{\prime}(M-R) / 15}
$$

where $k^{\prime}$ is the payload size used in the previous $M$ transmissions.

Combining equations (5)-(13), we can get $\eta_{R}(k)$ for DH and DM packets respectively as:

$$
\begin{gathered}
\eta_{R}(k)=\int_{0}^{1}\left[\frac{k(1-b)^{k}}{n} \times \frac{\left(\begin{array}{c}
M \\
R
\end{array}\right)\left(1-(1-b)^{k^{\prime}}\right)^{R}(1-b)^{k^{\prime}(M-R)}}{\int_{0}^{1}\left(\begin{array}{c}
M \\
R
\end{array}\right)\left(1-(1-b)^{k^{\prime}}\right)^{R}(1-b)^{k^{\prime}(M-R)} d b}\right] d b \\
\left.\frac{\left(\begin{array}{c}
M \\
R
\end{array}\right)\left(1-\left((1-b)^{15}+15 b(1-b)^{14}\right)^{k^{\prime} / 15}\right)^{R}\left((1-b)^{15}+15 b(1-b)^{14}\right)^{k^{\prime}(M-R) / 15}}{\int_{0}^{1}\left(\begin{array}{c}
M \\
R
\end{array}\right)\left(1-\left((1-b)^{15}+15 b(1-b)^{14}\right)^{k^{\prime} / 15}\right)^{R}\left((1-b)^{15}+15 b(1-b)^{14}\right)^{k^{\prime}(M-R) / 15} d b}\right] d b \\
\eta_{R}(k)=\int_{0}^{1}\left[\frac{k\left((1-b)^{15}+15 b(1-b)^{14}\right)^{k / 15}}{n} \times\right.
\end{gathered}
$$

where $\mathrm{n}=\mathrm{k}+126$.

It is now possible to choose the value of $k$, the payload length to be used in future transmissions, so that the throughput efficiency of the ARQ protocol is maximized. This can be done by choosing the value of $k$ that maximizes equation (14) or (15) for a value of $R$ that is equal to the number of retransmission requests that occurred during the previous $M$ transmissions using the payload size $k^{\prime}$.

\section{Simulation Results}

Usually, the solution way of the maximization problem for $\eta_{R}(k)$ in equation (7) or (8) is difficult; However, for specific values of $M, R$ and $k^{\prime}$ equation (7) or (8) can be solved numerically. An optimal value for $k$ can now be found using numerical search algorithms. Since the numerical evaluation of this integral is very intensive, a comprehensive search for the optimal value of $k$ is not practical. Instead, a restricted search using select values for $k$ can be performed. Such a search, for example, can consider values of $k$ that are a multiple of 100; thereby significantly reducing the complexity of the search. Such a restricted search has little impact on the performance of the protocol since values of $k$ that are within 100 bits of the optimal block size should result in near-optimal performance. Here, analysis of equation (14) or (15) is taken using Matlab7.0, where $k$ is always valued as the multiple of 100 and the pace of $b$ is 0.000001 .

In fig. 3, we plot the optimal payload size when a history of 50 previously transmitted 1500 bit packets payload is considered. As can be seen from the fig. 3 (a), for DH 
packets transmission, when the previous fifty transmissions resulted in no errors the payload length can be maximized to 2744 bits (the maximization throughput efficiency can be gotten at payload length of 3200 bits). When one and two errors occurred the payload length can be increased to 2100 and 1700 bits respectively. When three errors occurred the payload length can be kept at 1500 bits and when more than three errors occur the payload length is reduced. As depicted in fig. 3 (b), for DM packets transmission, when the previous fifty transmissions resulted in no errors the payload length can be maximized to be 2745 bits (the maximization throughput efficiency can be gotten at packet payload length of 4400 bits). When one, two and three errors occurred the payload length can be increased to 2500, 1900 and 1600 bits respectively and when more than three errors occur the payload length is reduced.

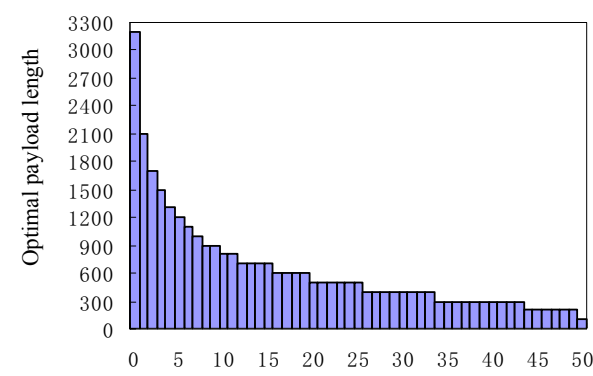

$\mathrm{R}$ (number of retransmission packets)

(a) DH packet

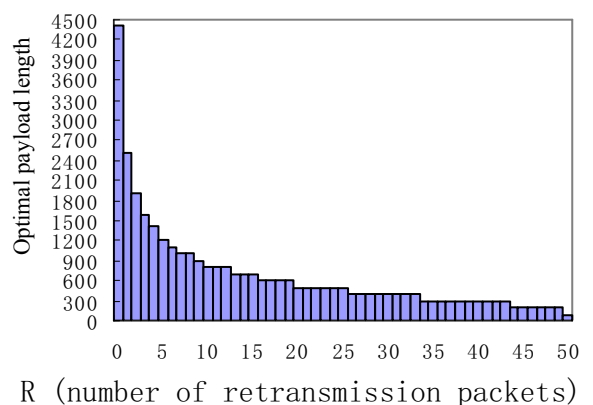

(b) DM packet

Fig. 3. Optimal packet size selection based on retransmission history

Let $k$ be the optimal packet size chosen for a given value of $R$ out of the last $M$ packet transmissions. The efficiency of the ARQ protocol with that value of $k$ can be computed according to equation (3) combined with equation (10) or (11). Then it can be averaged over the distribution of $R$ given $b$ to yield the performance of ARQ for a given value of $b$. Fig. 4 shows the mean throughput efficiency of ARQ with various values of $M$ and $b$, and a previous packet payload length of 1500 bits. As can be seen from the figure, whether it is DH or DM packets transmission, good performance is obtained with a history of just 75000 bits payload size (50 packets at packet payload size of 1500 bits). When $b$ is higher than $10^{-5}$, more of history is required to obtain a reasonable estimate of throughput efficiency for DM packets transmission; but for DH packets transmission, the situation is quite different, only few or the least history of packets transfer is required to obtain high throughput efficiency.

The throughput efficiency can be computed according to equation (3) using the optimal packet size obtained from the formula (4) under different channel BER. In the previous fifty packets transmission with payload length of 1500 bits, the mean throughput efficiency of DH is calculated based on the select optimizing packet size under different retransmission packets. Fig. 5 compares mean throughout efficiency of DH packets transmission based on retransmission history and the optimal packets transmission (opt) according to formula (4) for various $b$. As can be seen from the 


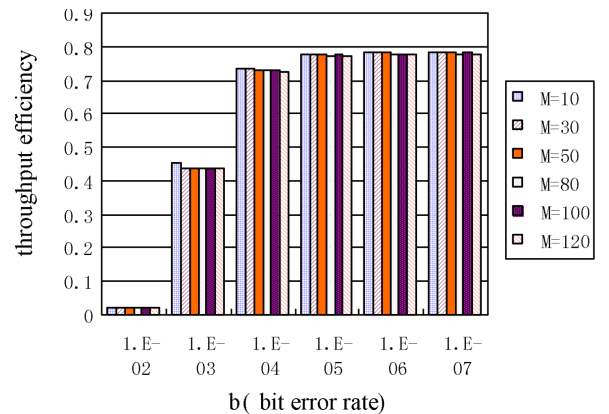

(a) $\mathrm{DH}$ packet

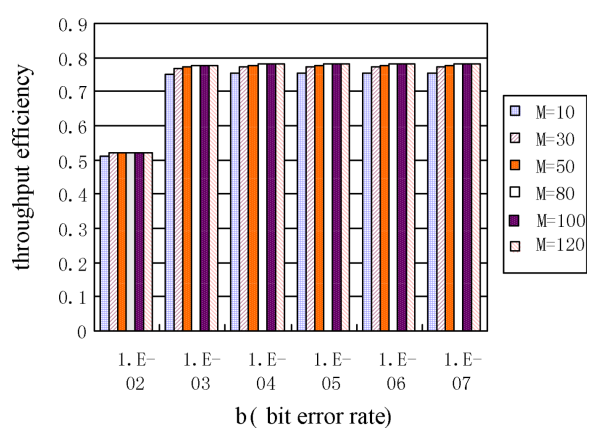

(b) DM packet

Fig. 4. Mean throughput efficiency of algorithm for various b

Fig. 5, both mean throughout efficiency increase against the decrease of $b$. The mean throughout efficiency of DH packets based on retransmission packets is always below to the optimal packets transmission (opt), but the difference is not so great.

Similarly, the mean throughput efficiency of DM is calculated based on the select optimizing packet size under different numbers of retransmission packets. Fig. 6 compares mean throughout efficiency of DM packets transmission based on retransmission history and the optimal packets transmission (opt) according to formula (4) for various $b$. As can be seen from the figure, both mean throughout efficiency increase by the decrease of $b$. The mean throughout efficiency of Bluetooth DM packets based on retransmission packets becomes mild while the $b$ is less than $10^{-3}$, which is close to 0.7773 . When $b$ is large than $10^{-3}$, the mean throughout efficiency of DM packets based on retransmission packets is larger than the optimal packets transmission (opt). Hence, the larger throughout efficiency can be gained by using DM packets transmission with ARQ protocol when the channel BER is high.

In the previous fifty packets transmission with payload length of 1500 bits, select optimizing packet size under different retransmission packets, fig. 7 compares mean throughout efficiency of ARQ during DH and DM packets transmission for various b.

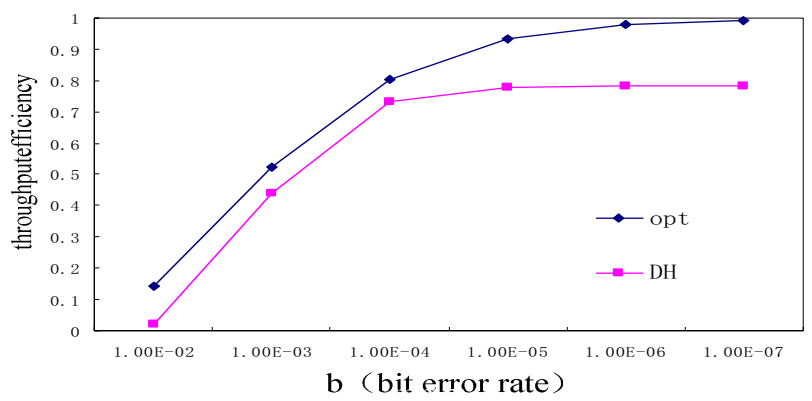

Fig. 5. Comparing mean throughput efficiency of DH and optimal packets transmission (opt) 


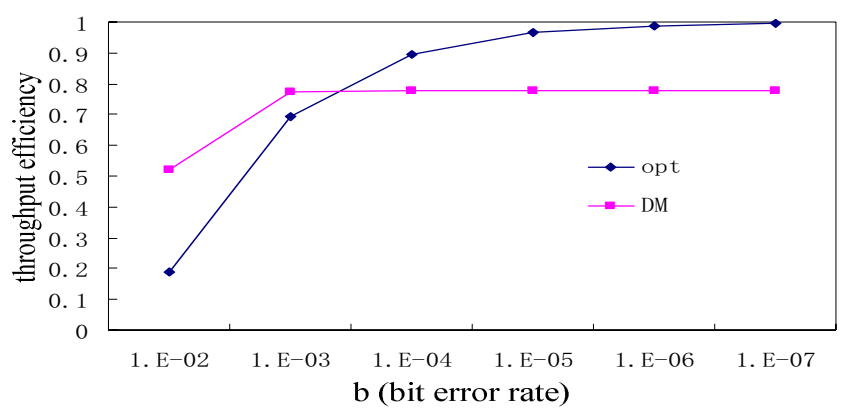

Fig. 6. Comparing mean throughput efficiency of DM and optimal packets transmission (opt)

It is important to note that the performance of ARQ is much more vulnerable to DH packets when $b$ is high. That is, when $b$ is high the use of DH or DM packet type can have a disastrous effect on the throughput efficiency, and DM packets transfer can produce higher throughput efficiency than DH packets. When $b$ is low, small variations in the throughput efficiency from the different bit error rate $b$, packet types (DH/DM). So in a high error rate environment, it is better to take DM packets as data transmission, which accords with the capability of DM to tolerate high transmission error rate. Oppositely, in a low error rate environment, it is better to take DH packets as data transmission, it is because not decoded by 2/3-FEC DH packets have relatively higher data transfer rate than DM packets data transmission.

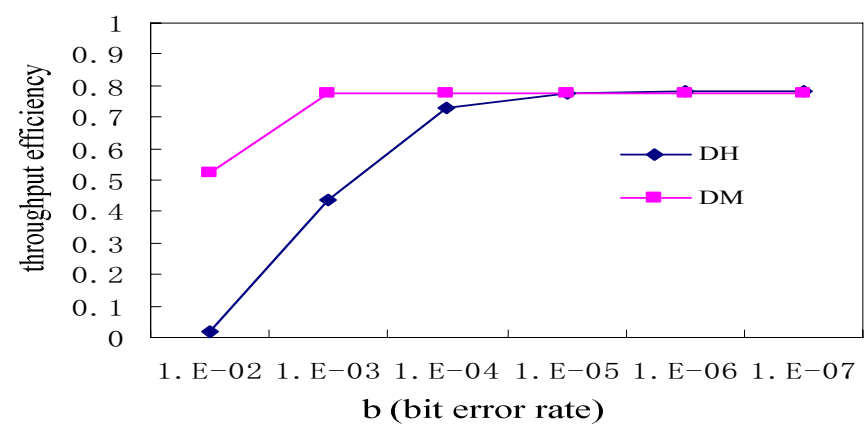

Fig. 7. Comparing mean throughput efficiency of DH and DM packets transmission

\section{Conclusion}

This paper introduces a method to select the optimal packet payload length used by Bluetooth ACL data link layer. The throughput efficiency of ARQ protocol is given based on retransmission history. So, given packet transmission record we can choose the packet size such that the expected throughput efficiency of the ARQ protocol is maximized under different channel BERs. Simulation results show that the method works very well even with a short observation history (50 packets at size of 1500 bits payload, total 75000 bits payload transfer). In a high error rate environment, it is 
better to take DM packets as data transmission; but in a low error rate environment, it is better to take DH packets as data transmission to improve the data transfer rate.

\section{References}

1. Bluetooth V1.1 Core Specifications, http: / /www. bluetooth. org

2. Harrtsen, J.: The bluetooth radio system. IEEE Personal Communicatios 7(1), 28-36 (2000)

3. Modiano, E.: An adaptive algorithm for optimizing the packet size used in wireless ARQ protocols. Wireless Networks 5, 279-286 (1999)

4. Chen, L.J., Kapoor, R., Sanadidi, M.Y., Gerla, M.: Enhancing bluetooth TCP throughput via link layer packet adaptation. In: Proc. of the 2004 IEEE International Conference on Communications (ICC 2004), pp. 4012-4016. IEEE Press, Paris (2004)

5. Valenti, M.C., Robert, M., Reed, J.H.: On the throughput of bluetooth data transmissions. In: Proc. of the IEEE Wireless Commun. and Networking Conf., pp. 119-123. IEEE Press, Orlando (2002)

6. Sarkar, S.: Optimal Communication in Bluetooth Piconets. IEEE Transactions on Vehicular Technology 54(2), 709-721 (2005)

7. Turin, W.: Throughput analysis of the Go-Back-N protocol in fading radio channels. IEEE Journal on Selected Areas in Communications 17(5), 881-887 (1999)

8. Pribylov, V.P., Chernetsky, G.A.: Throughput efficiency of automatic repeat request algorithm with selective reject in communication links with great signal propagation delay. In: Proc. of the 3-rd IEEE-Russia Conferences Microwave Electronics: Measurements, Identification (MEMIA 2001), pp. 202-205. IEEE Press, Novosibirsk (2001)

9. Jesung, J., Yujin, L., Yongsuk, K., Joong, S.M.: An adaptive segmentation scheme for the Bluetooth-based wireless channel. In: Proc. of the 10th International Conference on Computer communications and networks, pp. 440-445. IEEE Press, Scottsdale (2001) 